\title{
La dinámica de las firmas: un análisis desde la economía del conocimiento.
}

\author{
Duvan Emilio Ramirez Ospinal
}

Resumen

Este documento hace un análisis de la transformación estructural de dos sectores de la economía; para su desarrollo se hace un recorrido por la construcción teórica que diversos autores han realizado sobre conceptos como la sociedad del conocimiento, sociedad de la información, la sociedad en red, la economía del conocimiento y cómo influye en las dinámicas productivas de los territorios y de las firmas, contiene también un análisis de los indicadores pertinentes para determinar si hay un avance hacia una transformación estructural que evidencie el acercamiento de las Pequeñas y Medianas Empresas (PYMES) con la innovación tecnológica, las Tecnologías de Información y la Comunicación (TIC's), la Investigación y Desarrollo en los procesos y los productos de las empresas. También se hace referencia al trabajo de campo elaborado con las pymes de los sectores alimentos (biotecnología) y textil-confección (diseño) en Medellín-Valle del Aburrá y Manizales- Villamaría. Se presenta una nota metodológica, describiendo el proceso, el enfoque, los métodos y las herramientas utilizadas para el análisis de la información, seguido de la descripción estadística y análisis de los factores más representativos en dichas pymes con referencia a la transformación estructural basada en la economía del conocimiento.

Palabras clave: economía del conocimiento, redes, productividad, sociedad en red, sociedad del conocimiento, innovación, gestión del conocimiento, firmas.

\section{Abstract}

This paper makes an analysis of the structural transformation of two economy sectors; for its development a theoretical construction is made that different authors have made about concepts like the society of knowledge, society of the information, the net society, the economy knowledge and how is the influence on the productive dynamics of the territories and the firms, this paper also contains an analysis of the appropriate indicators to determinate if there is an advance for a structural transformation that shows the approach of small and medium firms for technological innovation, the information and communication technologies, the investigation and development in the productive process in the companies. It makes reference to the research with the small and medium firms of the food's (biotechnology) confection- textile's sectors (design) in MedellinValle de Aburra and Manizales-Villamaría. It shows a methodology note, describing the process, the focus, the methods and the tools used to the information analysis, followed by the statistical description and the most representative factors in those small and medium firms concerning the structural transformation based on the knowledge economy.

Keywords: knowledge economy, nets, productivity, society in net, knowledge society, innovation, knowledge management, firms.

\footnotetext{
${ }^{1}$ Economista de la Universidad de Manizales; Especialista en Mercadeo de la Universidad EAFIT. Magister en Gerencia del Talento Humano de la Universidad de Manizales; Candidato a Doctor en Administración de la Universidad Andina Simón Bolívar sede Ecuador. Profesión: Docente investigador Facultad de Mercadeo Nacional e Internacional Universidad De Manizales. Ubicación: Universidad de Manizales, Facultad de Mercadeo Internacional. Email: merca2@umanizales.edu.co; duvramirez@hotmail.com

Artigo enviado em outubro e aceito em dezembro de 2008.
} 


\section{Introducción.}

Este artículo es un producto de la investigación denominada "TRANSFORMACIÓN ESTRUCTURAL DE LOS SECTORES ALIMENTOS (BIOTECNOLOGÍA) Y CONFECCIONES (DISEÑO) EN MEDELLÍNVALLE DE ABURRÁ Y MANIZALES-VILLAMARÍA EN EL PERÍODO 1990-2004: ANÁLISIS DESDE LA ECONOMÍA DEL CONOCIMIENTO" Desarrollada por los grupos de investigación "Estudios Sectoriales y Territoriales" -ESYT- de la Universidad EAFIT y un grupo de investigadores de la Universidad de Manizales, en la República de Colombia.

La investigación se ha centrado en el análisis de la formación de competencias empresariales para la dinámica de la economía del conocimiento y en la identificación de las interacciones entre factores geográficos y políticos de organización industrial de los sectores alimentos y confecciones. Estos factores influyen en la dinámica de las $\mathrm{PYMES}^{2}$ en la incorporación de innovaciones ${ }^{3}$, y en su distribución en el territorio, que son consideradas dimensiones fundamentales para el análisis de la dinámica de las firmas. Se trata de un trabajo investigativo desarrollado en la búsqueda de resultados que propicien el análisis de los sistemas de conocimiento, innovación en los sectores alimentos y confecciones en dos regiones colombianas: ManizalesVillamaría y Medellín-Valle de Aburrá, orientados a la formulación de políticas sectoriales e influir en las políticas gubernamentales para estimular las actuaciones de los agentes privados.

Los sectores económicos alimentos y textil-confección han sido tradicionales en la actividad económica de las regiones tomadas como referente; además de generar un número importante de empleos y de impacto económico en el territorio. Es por esto que se hace interesante mirar las transformaciones estructurales y cómo se han adaptado los sectores a ellas, si proporcionan desarrollo al territorio, si han integrado nuevas visiones desde la economía del conocimiento.

Uno de los objetivos de la investigación, es identificar los elementos que evidencian las transformaciones estructurales en los sectores confecciones y alimentos; a partir del análisis de este proceso en las pymes ubicadas en las regiones señaladas. Con referencia a la apropiación de tecnología e innovación, se tuvieron en cuenta elementos como la historia empresarial en la cual se pudo observar la evolución que han tenido las empresas y de esta manera caracterizar el origen del cambio en los sectores. Otro objetivo estuvo orientado a generar espacios e indicar las vías que se deben usar para sensibilizar a policy makers, empresas, representantes de ONG's, gremios productivos y funcionarios públicos sobre la necesidad de establecer políticas focalizadas sectorialmente con lógica territorial y desde las especificidades y heterogeneidades productivas.

Los procesos de innovación y apropiación de la tecnología describen la manera como son insertados los nuevos elementos productivos que tienen que ver con el manejo de la información y su aprovechamiento para generar desarrollo empresarial y sectorial con base en el nuevo conocimiento; las redes industriales que tienen que ver con las relaciones que se establecen en el territorio, en el sector y si estas determinan o no su dinámica de

\footnotetext{
${ }^{2}$ Las pequeñas y medianas empresas -PyMEs-, a través del tiempo, han emergido como una categoría intermedia que articula el mundo de la producción en torno a los procesos innovativos que se desprenden de la flexibilidad estructural de las firmas. Estas unidades productivas son hoy factor de crecimiento y dinamismo en el sistema económico. Asociación colombiana de pequeños industriales (ACOPI), señala que en 2003, las microempresas, pequeñas y medianas industrias (MiPyMEs) representaron el $96 \%$ de las empresas existentes en Colombia, contribuyendo con el $49.6 \%$ del empleo industrial, $25 \%$ del Producto interno bruto (PIB), $25 \%$ de las exportaciones totales y el $33 \%$ de las exportaciones no tradicionales. Además, representan $92 \%$ de los establecimientos industriales, generan $33 \%$ del total de valor agregado, el $31 \%$ de la inversión neta y el $45 \%$ del consumo industrial nacional.

${ }^{3}$ La innovación es esencialmente un fenómeno de desequilibrio - un salto en la oscuridad- que requiere capacidades que sólo tienen unos pocos (ELSTER, 2000) y la forma básica de innovación es cualitativa y discontinua: "Es aquella clase de cambio que surge desde dentro del sistema que desplaza su punto de equilibrio de tal manera que el nuevo cambio no puede alcanzarse desde el anterior mediante pasos infinitesimales" (SCHUMPETER, 1934) Además, las actividades asociadas a la tecnología de productos y/o procesos se definen como el conjunto de procedimientos científicos, tecnológicos, organizacionales, financieros y comerciales que concluyen, o deberían concluir, en la realización de productos 0 de procedimientos tecnológicos nuevos o considerablemente mejorados para una firma (NEFFA, 2000).
} 
estabilidad o crecimiento; el conocimiento y las competencias laborales, elementos que miden el impacto de los procesos de sistematización de conocimiento e información en el empleo y en el recurso humano; y, el desarrollo empresarial y regional que hace referencia al efecto que tiene en el territorio la visión del desarrollo desde la economía del conocimiento y desde la transformación estructural de los diferentes sectores económicos.

El enfoque metodológico adoptado es el empírico-analítico, que comprende un trabajo de campo, por medio del cual se pudo obtener información con base en cuestionarios aplicados a una muestra de empresas de estos sectores ubicadas en las regiones colombianas: Medellín-Valle del Aburrá y Manizales-Villamaría.

El proceso investigativo se desarrolló en tres fases así: 1. Revisión Bibliográfica que incluye el estudio conceptual de las categorías empleadas en el trabajo; revisión de las políticas de fomento de la ciencia y tecnología en la República de Colombia; revisión del marco normativo colombiano que tiene que ver con el fomento de la ciencia y la tecnología. 2) Diseño técnico; esta fase comprende la elaboración de herramientas para la recolección de información; diseño del cuestionario orientado a determinar el uso y apropiación de nuevas tecnologías en las empresas de las regiones de estudio. 3) Trabajo de Campo; en esta fase se realizó la selección de las empresas participantes en el proceso de generación de información; para el diligenciamiento del cuestionario se toma una muestra de pymes en las regiones de Manizales- Villamaría y Medellín- Valle del Aburrá y en los sectores alimentos y confección.

Para identificar estos elementos en las empresas objeto de estudio, se acudió al uso del cuestionario estructurado por módulos, a través del cual se pudo indagar sobre la historia y caracterización de la empresa, los procesos de innovación y apropiación de tecnología, la constitución o vinculación a redes empresariales, los conocimientos y competencias laborales desarrollados y el desarrollo empresarial de la región.

En la elaboración de los perfiles se utilizaron los estadísticos de frecuencia y las medidas de tendencia central; para un análisis más detallado de las respuestas abiertas y en profundidad dadas por los empresarios, se acudió al análisis textual (por medio del programa Atlas ti). Como una forma de lectura de los datos multivariados se utiliza el software para análisis estadístico de datos cuantitativos Spad, ésta es una herramienta para la sistematización de la información que permitió visualizar directamente la relación entre las categorías de análisis presentadas en la codificación obtenida por medio de los cuestionarios, permitiendo una lectura cuantitativa de los datos y poniendo en evidencia las tendencias.

Se realizo un análisis factorial el cual permitió identificar qué variables pertenecen al mismo grupo, cuáles acumulan el mayor peso sobre las otras y en qué grado lo hace. Por lo tanto reduce el número de variables y ayuda a localizar e identificar unidades o propiedades fundamentales en las que se basan pruebas y medidas. En resumen podría decirse que este método ayuda a verificar las expectativas teóricas.

\section{La sociedad de la información.}

Desde diversos enfoques, se está construyendo el paradigma informacional, autores como Yoneji Masuda ven a la sociedad de la información y del conocimiento como una "sociedad que crece y se desarrolla alrededor de la información y aporta a un florecimiento general de la actividad intelectual humana, en lugar de un aumento del consumo material" (VALLE, 2004, p.117).

Este paradigma se materializa en los sistemas de producción y se transforma por medio de tecnologías de la información. En tal sentido, si se le da dimensión a la forma de organización y productividad de la sociedad contemporánea, ésta no depende del "incremento cuantitativo de los factores de producción (capital, trabajo, recursos naturales), sino de la aplicación de conocimiento e información a la gestión, producción y distribución, tanto en los procesos como en los productos", cuyo núcleo de desarrollo según Castells (1998) es: la información como materia prima. 
Sin embargo, en una sociedad informatizada se presenta el problema del exceso de información que como sociedad genera. El desafío consiste en cómo convertir información en conocimiento útil, además de aprovechar el proceso de generación y apropiación del conocimiento para estimular procesos dinámicos de aprendizaje social, convirtiéndose este en factor de cambio para la sociedad, sus instituciones, o las empresas del sector productivo (CHAPARRO, 1998, p. 12).

Debido a su importancia, los procesos de aprendizaje social que la generación y aplicación (apropiación) del conocimiento pueden generar, se convierten en el eje central de una estrategia orientada hacia la construcción de una Sociedad del Conocimiento. Es este proceso el que lleva al desarrollo de lo que en la reciente literatura sobre el conocimiento y la innovación se denominan como sociedades del aprendizaje (learning societies), organizaciones que aprenden (learning organizations), y redes de aprendizaje (learning networks). Esto lleva a una concepción dinámica de la relación entre el conocimiento, el sujeto que conoce y el entorno en el cual el mismo actúa. Es a través de este proceso que el conocimiento hace posible que los miembros de una sociedad construyan su futuro y por lo tanto incidan en el devenir histórico de la misma (CHAPARRO, 1998)

La sociedad del conocimiento, no se limita a la revolución en las tecnologías de la información y comunicación ${ }^{4}$, ni al comercio electrónico o la posibilidad de trasmitir información a cualquier parte del globo en cuestión de segundos. La Economía del Conocimiento demanda nuevas competencias vinculadas con las necesidades de la sociedad, involucra la capacidad de aprendizaje de las instituciones, el sector productivo y académico, así como la generación de redes interinstitucionales para la solución de problemas y el uso intensivo del conocimiento a nivel societal. Las instituciones: su cantidad y calidad; las relaciones entre ellas; la consolidación de las redes institucionales; y, el tipo de organización que tienen, son factores que constituyen un actor importante en la acción económica y en la innovación, que son parte de la misma y no sólo un bastión o soporte de las firmas.

De esta forma, la transformación estructural de los sectores y regiones se refleja en las acciones dentro del proceso productivo de las empresas, pero también en la capacidad de generar procesos de innovación en la gestión y en la acumulación e intercambio de aprendizajes individuales y colectivos que hagan referencia a una nueva visión de la productividad en el territorio basado en el conocimiento.

La noción de sociedad del conocimiento fue introducida por Drucker a principios de los años 90 del siglo XX, a partir de sus análisis sobre la posibilidad de la aplicación del conocimiento para lograr nuevos avances en el propio conocimiento, lo cual lo convierte en motor de desarrollo al permitir una rápida adaptación a los cambios y facilitar una vía de solución a los problemas económicos. La noción de conocimiento se une así a la de información, concepto que ya se venía usando para designar al nuevo tipo de sociedad, con el fin de formar parte de procesos comunicativos, de intercambios simbólicos que tienen el efecto de reforzar algunas de las características más destacadas de lo que hoy se denomina sociedad del conocimiento.

David y Foray (2002) sostienen que aunque el conocimiento siempre ha estado presente en el proceso de producción, el desarrollo de las tecnologías de la información y la comunicación (TIC’s) está permitiendo el acceso a una abundante información, en condiciones inéditas, aumentando la potencia creativa de las interrelaciones, mejorando la gestión de bases de datos y posibilitando el desarrollo de sistemas descentralizados y de gran escala para la recopilación y cálculo de datos y de intercambio de los resultados.

En este contexto, es posible interpretar la Sociedad de la Información como una etapa previa a la conformación de la Sociedad del Conocimiento, en la que se aplican las TIC's a un sinnúmero de actividades, lo que constituye la base para el posterior desarrollo de la Sociedad del Conocimiento, que implica un cambio

\footnotetext{
${ }^{4}$ Las Tecnologías de la Información y las Comunicaciones -TIC- se definen como el conjunto de instrumentos, herramientas o medios de comunicación como la telefonía, los computadores, el correo electrónico y la Internet que permiten comunicarse entre sí a las personas u organizaciones.
} 
cualitativo en el uso y aplicación de la información para la generación de nuevos conocimientos, cambio basado en la educación y el aprendizaje. (BIANCO, et. al, 2002, p.9).

La sociedad de la información y del conocimiento se están empezando a conformar sobre la base de los cuatro grupos (PÉREZ, 2003, P.53): 1) Usuarios: son las personas y organizaciones que acceden a los contenidos a través de las infraestructuras. A su vez, dentro de los usuarios se distinguen tres tipos: ciudadanos, empresas y administraciones públicas; 2) Infraestructuras: son los medios técnicos que posibilitan el acceso remoto a los contenidos; 3) Contenidos: se llama de esta forma a la información, los productos y los servicios a los que se puede acceder sin necesidad de desplazarse a un lugar determinado; y, 4) Entorno: son factores o agentes de tipo social o económico, que afectan a la orientación y al ritmo de implantación de la sociedad de la información.

La principal distinción entre Sociedad del Conocimiento y Sociedad de la Información es el uso, apropiación y aplicación que se le puede dar a la información y al conocimiento, con lo que queda claro que no se trata de un problema tecnológico. En este sentido, Chaparro (1998) define la sociedad del conocimiento como "una sociedad con capacidad para generar conocimiento sobre su realidad y su entorno, y con capacidad para utilizar dicho conocimiento en el proceso de concebir, forjar y construir su futuro. De esta forma, el conocimiento se convierte no solo en instrumento para explicar y comprender la realidad, sino también en motor de desarrollo y en factor dinamizador del cambio social".

De otro lado, el auge del modelo exportador y la globalización de la economía, han generado nuevas relaciones productivas aumentando sus capacidades de producción tanto en capital como en trabajo, teniendo en cuenta las tecnologías como forma de acumulación y difusión del conocimiento para la transformación industrial. Esto supone que la sociedad es capaz de constituirse en red, articulando los individuos en torno a instituciones y sectores que permiten superar las fronteras de la innovación y el desarrollo a nivel global y local (CARDONA, 2003, p.15).

La interconexión de empresas o, sin ir más lejos, la crisis de la gran empresa vertical y el desarrollo de las redes empresariales, son ejemplos de éste fenómeno. Sobre el particular, Castells (1996) afirma que las nuevas trayectorias organizativas no son una consecuencia mecánica del cambio tecnológico, sino que, por el contrario, algunas de ellas precedían al surgimiento de las nuevas tecnologías de la información. Pero de lo que no cabe duda es que las nuevas tecnologías de la información tienen una incidencia directa en la gestión empresarial y en las competencias laborales individuales y que de estos sumados a la flexibilidad de actuación, depende en gran medida el éxito o fracaso en el mercado. (CANO, 2006, p. 8).

De esta forma, la capacidad de generar competitividad y productividad en las firmas radica en las redes de intercambio de información, en la interacción con sus proveedores, con sus clientes y con su mercado potencial y natural y en la imperante condición de la innovación. Esa sociedad es una estructura construida en torno a redes de información que constituyen la forma organizativa actual. Finalmente, y desde una visión prospectiva, Chaparro (1998, p.63) plantea que "los sectores productivos juegan un papel crucial en la construcción de la sociedad futura del conocimiento, por su enorme dinámica para generar innovaciones, concebir productos y procesos, y generar empleo. La nueva empresa del siglo XXI es un ente social que utiliza intensivamente el conocimiento a fin de responder a las expectativas de una sociedad informatizada, con mayor capacidad de participación del trabajo"; de esta forma, la economía se convierte en una "economía del conocimiento"

\section{La economía del conocimiento}

La "economía del saber" surge cuando un conjunto de personas co-producen (es decir, producen e intercambian) conocimientos con la ayuda de tecnologías de la información y de la comunicación. Existen, por tanto, tres elementos: i) la producción y la reproducción de nuevos conocimientos son asumidas por un conjunto de miembros de la comunidad, ii) la comunidad crea un "espacio público" de intercambio, de circulación de los saberes, y iii) el empleo de nuevas tecnologías de información y comunicación es intenso para codificar y transmitir los conocimientos nuevos (DAVID y FORAY, 2002) 
Para Castells (2000), la economía del conocimiento es una economía que está centrada en el conocimiento y en la información como bases de producción, como bases de la productividad y bases de la competitividad, tanto para empresas como para regiones, ciudades y países. Esto se dice fácil, pero tiene grandes consecuencias, porque el tratar sobre la forma cómo se genera productividad quiere decir cómo se genera riqueza, que en el fondo es la base material para lo que luego se pueda hacer.

La economía del conocimiento es una economía que funciona en redes, en redes descentralizadas dentro de la empresa, en redes entre empresas, y en redes entre las empresas y sus redes de pequeñas y medias empresas subsidiarias. Es esta economía en red la que permite una extraordinaria flexibilidad y adaptabilidad. Es, por tanto, una economía informacional, una economía global y una economía organizada en red, y ninguno de esos factores puede funcionar sin el otro.

El recurso más escaso y, a la vez, fundamental en una economía basada en el conocimiento es la capacidad que toda organización tiene de generar conocimiento. El conocimiento se ha convertido por tanto, en el motor de crecimiento económico y de la mejora de la productividad y, por tanto, en factor productivo y elemento diferenciador. (BARCELÓ, 2001, p.13). El nuevo papel jugado por el conocimiento está originando que los países vean transformar sus economías en "economías basadas en el conocimiento". La creciente importancia de la economía basada en el conocimiento es posible gracias a la confluencia de una serie de factores que están posibilitando este movimiento: el crecimiento de las tecnologías de la información y de las comunicaciones, la globalización, los cambios de la demanda y los avances científicos y tecnológicos. Estos factores interfieren unos sobre otros promoviendo un proceso de modificación continuo.

La innovación, es decir, la aplicación del conocimiento para producir nuevo conocimiento no es, como tanta gente cree, cuestión de "inspiración", ni que la realicen individuos aislados en el garaje de su casa. Requiere esfuerzo sistemático y un alto grado de organización. Pero también requiere tanto de descentralización como diversidad, es decir, lo contrario de planificación central y centralización (DRUCKER, 1994, p. 207)

La productividad del conocimiento es, cada vez más, el factor determinante en la posición competitiva de un país, una industria, una compañía. Con respecto al conocimiento, ningún país, ninguna industria, ninguna compañía tiene ninguna ventaja o desventaja "natural". La única ventaja posible estará en cuánto pueda obtener con el conocimiento universalmente disponible. Lo único que va a tener importancia en la economía nacional, lo mismo que la internacional, es el rendimiento de la administración en hacer productivo el conocimiento (DRUCKER, 1994, p. 211).

La economía del conocimiento, según Del Valle (2006), es el estudio de los procesos de creación, apropiación, transformación y difusión de habilidades y destrezas que permitan la solución de problemas económicos. Una economía basada en el conocimiento es una economía en la cual la creación, distribución y uso del conocimiento es el mayor propulsor de crecimiento, riqueza y empleo.

Dahlman y Andersson (2000) plantean que una economía basada en el conocimiento es una en la cual "el conocimiento (codificado y tácito) es creado, adquirido, transmitido y utilizado más efectivamente por empresas, organizaciones, individuos y comunidades para un mayor resultado económico y social".

Desde otra postura pero complementaria a la anterior, se plantea que "en las economías basadas en el conocimiento, la capacidad de gestión y de intermediación (distribución) de dicho conocimiento juega un papel vital. En el desarrollo de esta capacidad, las redes científicas, o redes del conocimiento, en las que participan tanto generadores del conocimiento (investigadores) como usuarios del mismo, se están constituyendo en la espina dorsal de las nuevas sociedades" (OCDE, 1996 en CHAPARRO, 1998, p. 6).

En general es difícil trazar una línea clara de separación entre el conocimiento y la información, y lo mismo ocurre entre la información y los datos. Sin embargo, no por ello la distinción es menos importante. En muchas circunstancias, los términos "conocimiento" e "información" se emplean como sinónimos. Quizás es por esto por lo que no siempre se distingue claramente entre "Sociedad del Conocimiento" y "Sociedad de la Información". De la misma manera, si no se distinguen estos dos conceptos es imposible medir el impacto o la 
contribución del conocimiento en la generación de valor y crecimiento económico (BIANCO, et. Al., 2002, p. $8)$.

Según Hansson (2002) "el conocimiento es un concepto complejo. En primer lugar, el conocimiento es una especie de creencia. Y en segundo lugar, lo que se considera conocimiento tiene que estar justificado. Por lo tanto, el conocimiento tiene elementos tanto subjetivos como objetivos. El conocimiento lo podemos definir como una creencia verdadera y justificada. Esta definición se puede complicar incluyendo el tema de la asimilación cognitiva. Los datos tienen que ser asimilables para que se puedan calificar de información, y cognitivamente asimilados para que se puedan calificar de conocimiento".

El concepto del conocimiento es mucho más amplio que el de información. Los componentes fundamentales del Know What y del Know why se pueden calificar como información. Mientras que el know how y el Know who el conocimiento tácito es mayor y mucho más difícil de codificar y medir. (BOSCHERINI et. al., 2003, p. $15)$.

El know what -el saber qué- se refiere al conocimiento de hechos. En estos casos, el conocimiento se aproxima a lo que habitualmente se llama información y se puede descomponer en bits. EL know why -el saber porquése refiere a lo que hay que saber a cerca de los principios y leyes de la Naturaleza. El desarrollo tecnológico de la mayoría de los productos y servicios se apoya sobre esta información. La producción y reproducción del Know why se hace en las universidades y en los laboratorios de investigación científica. El know how -saber cómo- se refiere a la habilidad o capacidad, para hacer algo. Es lo que aplica un empresario cuando busca clientes para un nuevo producto o un gerente de recursos humanos cuando selecciona personal o lo entrena, también es el caso del trabajador que opera una innovación.

El desarrollo de las tecnologías de la información puede verse como una respuesta a la necesidad de máquina complicada. El know how habitualmente se desarrolla y conserva dentro de una empresa. Por todo esto, el know who toma cada día más importancia, ya que implica saber quien sabe qué hacer y quien sabe cómo hacerlo. El "saber quien" implica el desarrollo de relaciones sociales que permitan acceder a los expertos y especialistas y saber como se debe usar eficientemente lo que ellos saben.

Es fácil deducir que cada saber se consigue a través de diferentes mecanismos: se puede adquirir know what y know why leyendo libros, escuchando conferencias o consultando bases de datos, mientras que los otros dos, se consiguen sólo con la experiencia. El know how es el que transmite el maestro al aprendiz, mientras que el know who es fruto de la experiencia social sobre todo, en ambientes educativos, y es muy difícil de transferir a través de los mecanismos formales de información.

Este es uno de los motivos por el que algunas empresas encargan investigaciones científicas, ya que así, acceden a las redes de especialistas académicos, que son críticas para mantener su capacidad de manipular con mayor eficiencia las porciones del conocimiento que se refieren al know what y al know why. Pero también, la existencia de las TIC's y de las infraestructuras de telecomunicaciones da un fuerte impulso al proceso de codificación de algunos tipos de conocimientos, ya que todo conocimiento que pueda ser codificado y reducido a información podrá ser transmitido con otros mínimos.

\section{Indicadores de la sociedad del conocimiento}

Los indicadores que se diseñen para medir una Sociedad del Conocimiento deben dar cuenta de los niveles de aprendizaje y de creación de capital social, a sabiendas de las dificultades que presenta medir procesos de aprendizaje acumulativos (BIANCO, et. al., 2002, p. 12). Esta dificultad debe ser superada por medio de nuevas investigaciones que permitan determinar la mejor forma de medir el nivel de penetración de la Sociedad del Conocimiento en una economía o en una nación.

Uno de los problemas es cómo medir el aporte del conocimiento al crecimiento económico y la productividad tanto de las regiones como de las organizaciones, con miras a distinguir el conocimiento de otros factores de 
producción. Howitt (1996) afirma que "si bien se han hecho progresos de modelar el conocimiento en un nivel teórico, menor progreso se ha hecho en el nivel empírico. Si el conocimiento es efectivamente diferente de otros bienes, por lo tanto debe ser medido de manera diferente, y su relación con el sistema de precios debe ser igualmente diferente de las de otros bienes". (BIANCO, et. al., 2002, p. 12).

$\mathrm{Al}$ igual que con los indicadores de innovación, se está frente al problema de cómo medir no sólo el stock (conocimientos, capacidades, etc.) sino procesos y flujos. Así como en innovación no sólo interesan los resultados (innovaciones) sino también las actividades y esfuerzos, en lo que tiene que ver con la Sociedad del Conocimiento se debe intentar captar las interacciones (entre individuo y sociedad; entre los integrantes de una organización, empresa o institución; entre una organización y otros componentes del sistema) que tienen la potencialidad de incrementar los niveles de conocimiento individual y colectivo.

\section{Indicadores de la economía del conocimiento}

La medición en la economía basada en el conocimiento supone la evaluación de la producción, distribución y uso del conocimiento. Sin embargo, la medición del conocimiento no resulta fácil, entre otras cosas, por las propias características que le hacen diferente del resto de bienes y servicios, lo cual dificulta e incluso cuestiona la validez de los indicadores tradicionales.

Howitt (1996) señala algunos de los aspectos que diferencian la medición de los bienes de capital de la medición del conocimiento (MUNSTER, 2007, p.3): 1) Las dificultades relacionadas con los insumos (inputs); 2) la evaluación de los resultados (outputs), 3) la cuantificación de cambios fundamentalmente en la calidad de los bienes y servicios.

Aún cuando se reconocen dificultades en la medición o utilización de medidas cardinales del "conocimiento" en los modelos formales económicos (STEEDMAN, 2001) se reconocen ciertos indicadores Proxy. Estos se agrupan generalmente en cuatro categorías principales (DEL VALLE, 2006, p. 7): 1) Creación de conocimiento, 2) Adquisición/ transferencia de conocimiento, 3) Difusión del conocimiento, y, 4) Aplicación del conocimiento. 
Indicadores proxy de la innovación

\begin{tabular}{|c|c|c|}
\hline & Indicadores proxi & Aspecto de conocimiento a medir \\
\hline \multirow{3}{*}{ 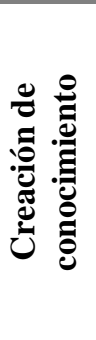 } & Gasto en I \& D como & Intensidad de la I \& D realizada en la economía \\
\hline & Investigadores per cápita & $\begin{array}{l}\text { Disponibilidad de recursos humanos necesarios } \\
\text { para llevar a cabo actividades de I \& D }\end{array}$ \\
\hline & Patentes Per cápita & \\
\hline \multirow{3}{*}{ 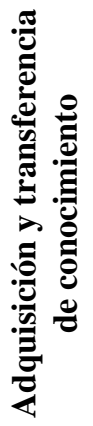 } & $\begin{array}{l}\text { Porcentaje de las } \\
\text { importaciones de contenido } \\
\text { tecnológico }\end{array}$ & $\begin{array}{l}\text { Contenido de conocimiento tecnológico incluido } \\
\text { en las importaciones }\end{array}$ \\
\hline & $\begin{array}{l}\text { Cantidad de oficinas } \\
\text { centrales y regionales de } \\
\text { las empresas del país }\end{array}$ & $\begin{array}{l}\text { Cantidad de empresas con conocimiento } \\
\text { especializado establecido por las campañas } \\
\text { multinacionales y regionales }\end{array}$ \\
\hline & $\begin{array}{l}\text { Número de empresas en el } \\
\text { sector de servicios } \\
\text { intensivos del } \\
\text { conocimiento }\end{array}$ & $\begin{array}{l}\text { Servicios prestados a industrias intensivas en } \\
\text { conocimiento, fomentando el proceso creativo y } \\
\text { de adquisición y transferencia de conocimiento } \\
\text { por actividades y rutinas }\end{array}$ \\
\hline \multirow{3}{*}{ 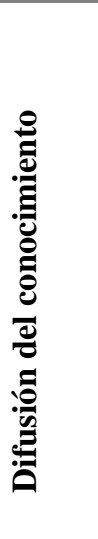 } & $\begin{array}{l}\text { Gasto en tecnologías de la } \\
\text { información y } \\
\text { comunicación TIC's }\end{array}$ & $\begin{array}{l}\text { Intensidad de recursos de infraestructura de } \\
\text { información y comunicación disponibles para la } \\
\text { transferencia y difusión de conocimiento }\end{array}$ \\
\hline & $\begin{array}{l}\text { Costos de acceso al } \\
\text { Internet como porcentaje } \\
\text { de PIB per cápita }\end{array}$ & $\begin{array}{l}\text { Accesibilidad de servicios de TIC's como } \\
\text { determinantes de su uso }\end{array}$ \\
\hline & $\begin{array}{l}\text { Porcentaje de la fuerza } \\
\text { laboral con -por lo menos- } \\
\text { educación secundaria }\end{array}$ & $\begin{array}{l}\text { Destrezas lingüísticas y tecnológicas necesarias } \\
\text { para hacer uso de las TIC's }\end{array}$ \\
\hline \multirow{3}{*}{ 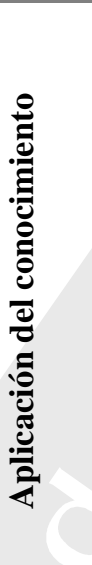 } & $\begin{array}{l}\text { Porcentaje de la fuerza } \\
\text { laboral con educación } \\
\text { universitaria }\end{array}$ & $\begin{array}{l}\text { Capacidad de la fuerza laboral de buscar, } \\
\text { procesar y usar información relevante }\end{array}$ \\
\hline & $\begin{array}{l}\text { Porcentaje de trabajadores } \\
\text { del conocimiento en la } \\
\text { fuerza laboral }\end{array}$ & $\begin{array}{l}\text { Cantidad de empleos que requieren y promueven } \\
\text { la aplicación de conocimiento }\end{array}$ \\
\hline & $\begin{array}{l}\text { Rango de conducta } \\
\text { empresarial } \\
\text { (entrepreneurship) }\end{array}$ & $\begin{array}{l}\text { Capacidad de la economía de crear nuevos } \\
\text { modelos empresariales que propendan a la } \\
\text { creación, adquisición, difusión y aplicación de } \\
\text { nuevas ideas y procesos }\end{array}$ \\
\hline
\end{tabular}

Fuente: Heng, Toh Mun, Tang Hsiu Chin \& Adrian Choo, 2002, en Del Valle 2006: 7) 


\section{Evidencia empírica (descriptiva y factorial)}

\section{Módulo historia empresarial}

Para crear un perfil de las empresas se acudió al módulo historia empresarial que tenía como objetivos identificar los elementos representativos de las empresas en su origen, si responden a iniciativas de los empresarios, a las políticas empresariales, a necesidades del mercado, además de determinar dentro del presente estudio, el cambio en la apropiación y uso de tecnologías por las empresas entrevistadas.

Otro de los elementos que hacen referencia al surgimiento empresarial es el capital con el que cuentan para distribuirlo en compra de maquinaria, localización, servicios, transporte, compra de materias primas, pago de proveedores, pago de nómina, entre otros gastos. La mayoría de empresas entrevistadas acuden al ahorro familiar (55.56\%) o créditos formales (33.33\%), esto demuestra que las posibilidades de crear empresa están ligadas directamente a las redes informales que se crean desde la familia y a las oportunidades individuales que el empresario posea para realizar un préstamo en una entidad financiera. Son pocas las empresas que acuden al mercado de valores, a un crédito de fomento o incubadora (16.67\%) unas por desconocimiento y otras por desinterés (manifestadas en las entrevistas realizadas).

El uso de tecnologías para el funcionamiento de las empresas entrevistadas ha sido encaminado hacia la comunicación y la información en su origen, esto se manifiesta en que el 50\% de las empresas entrevistadas utilizaban fax, el $47 \%$ teléfono celular, el $25 \%$ correo electrónico y maquinaria especial para la producción de alta tecnología. La importancia atribuida a la necesidad de crear redes de comunicación e información que mejoren los procesos productivos va creciendo; en la actualidad las mismas tecnologías en un porcentaje más elevado (fax $86 \%$, teléfono celular 98\%, correo electrónico 100\%), sin embargo, al inicio observamos que son pocos los que poseen software especializados para producción y para el tratamiento de la información, unos por desconocimiento y otros por los costos que implica la actualización de toda la planta. Pero a medida que va creciendo la empresa, crece la necesidad de adaptarse a las nuevas tecnologías, en especial las que hacen más eficiente el proceso de trabajo.

Las tecnologías de información y comunicación a las cuales acceden recién fundada la empresa son elementos básicos y necesarios para establecer un mínimo de interlocución con los proveedores o clientes. El acercamiento a la innovación en este ámbito no es muy referido, las empresas se centran más en la innovación de procesos y productos, mejoramiento de maquinaria para la producción. La ubicación de las ventas se enfoca hacia un mercado local, por lo que la comunicación es más directa y pueden darse procesos de aprendizaje y fortalecimiento de redes acordes a las necesidades del territorio. Esta variable es de suma importancia, ya que los sectores a los que se hace mención son tradicionales y reconocidos por la localidad, esto determina las acciones empresariales y la dinámica productiva del territorio.

\section{Módulo Procesos de Innovación y apropiación de lãs TICS's}

El presente modulo tiene como finalidad describir la innovación y los procesos de apropiación y transferencia de tecnologías realizado en las pymes. Para esto, se realizaron preguntas a cerca de las transformaciones que ha tenido la empresa en materia de tecnología y su aprovechamiento dentro de los procesos de trabajo y comunicación.

La mayoría de los empresarios entrevistados (92\%), han introducido cambios referentes a procesos de innovación empresarial y transferencia de tecnologías, sobresaliendo dentro de ellos la introducción de una mejora tecnológica en el proceso de producción (72.22\%) y la introducción de un nuevo producto o una nueva calidad de un producto (66.44\%), esto indica que la innovación va directamente relacionada con el proceso de trabajo y al producto. Aunque también se observa una notoria importancia del mercadeo y las ventas y desarrollo de planificación y estrategias. 
Los cambios introducidos en la empresa han tenido diversos resultados sobresaliendo entre ellos el aumento de la productividad (72.22\%) y un mejor ajuste a especificaciones de los clientes en calidad (58.33\%). La importancia que le atribuyen los empresarios a la productividad es alta y las acciones que tengan que ver con procesos de innovación van encaminadas a aumentarla y a tener una buena relación con sus clientes a partir de una mejora en el producto o en la calidad de su producto (44.44\%). Además, el principal objetivo explícito de la innovación empresarial es aumentar la participación en el mercado, y esto requiere de acciones que tengan en cuenta los procesos internos que mejoren 1) la calidad y 2) las redes productivas.

El impacto de la transferencia de tecnologías e innovación sobre el empleo es considerable, la mayoría de empresas entrevistadas (72\%) afirman que debido a estos procesos aumentaron el empleo. Las características de éste empleo tienen que ver con una mayor calificación y competencias laborales, que están relacionados con manejo de tecnologías en procesos de producción, además, los puestos administrativos y de gestión requieren de conocimientos en TIC's y de constante actualización de información referente no solo a la industria, sino al mercadeo (información tomada de los cuestionarios).

Pero no sólo se contrata desde fuera el personal capacitado para tales fines, las empresas han realizado propuestas de mejoramiento del producto (75\%), ajuste en la organización del trabajo y manejo de mayores volúmenes de producción (72.22\%) como acciones requeridas en la empresa para la adaptación a cambios tecnológicos.

Las empresas entrevistadas afirman recibir apoyo de las instituciones públicas para procesos de innovación y transferencia tecnológica, sin embargo, son bajos los porcentajes de apoyo, los procesos de transferencia de tecnología lo hacen de manera individual, es decir, cada empresa importa, compra maquinaria y a su vez se capacitan de manera independiente. La institución que más se ha dado en reconocimiento de este tipo de acciones es el Servicio Nacional de Aprendizaje (SENA) (30.58\%), la Cámara de Comercio y Proexport (con $22.22 \%)$.

Un porcentaje considerable de las empresas (42\%) afirman comprar y renovar maquinaria de uno a tres años, seguido de 4 a 6 años (con un 28\%), de 7 a 10 años (17\%). Esto indica niveles de actualización de los procesos industriales para tener una mayor productividad y adaptación en el sector y en el mercado. Los mayores problemas para la compra de maquinaria son la financiación (con un 62\%), los costos en las importaciones $(28 \%)$ e información de la maquinaria (10\%).

Dentro de los mecanismos de financiamiento para acceder al tipo de maquinaria requerida se tiene, en primer lugar (28\%) la compra directa, seguido de crédito formal (27\%) y leasing (25\%), en últimas instancias han acudido al crédito de proveedores $(12 \%)$ y crédito de fomento o incubadora (8\%). Son pocas las empresas que han accedido a créditos por parte de instituciones o programas de apoyo sectorial o regional, los empresarios han manifestado llevar procesos independientes ligados a los aprendizajes propios de la empresa.

Los medios de información utilizados para enterarse de las nuevas tecnologías requeridas para la producción son, en primer lugar, las ferias y exposiciones (21\%), seguido de las revistas especializadas (19\%) y la información de clientes y proveedores (18\%). Esto puede sugerir la necesidad de crear más espacios de comunicación entre empresarios, las ferias son un escenario donde se intercambia no sólo información sobre la maquinaria utilizada sino que en él se crean contactos, redes para la socialización de conocimientos a nivel sectorial.

Los productos que crean las empresas pasan por diversas etapas, desde el diseño e introducción al mercado hasta la aceptación, venta y consumo, una gran parte de los empresarios consideran que su principal producto es maduro (39\%), es decir, que ha tenido un proceso de aceptación en el mercado y que tiene un tiempo de consolidación considerable. Otro grupo (25\%) considera que es novedoso, que apenas está insertándose en el mercado con un impacto fuerte por su innovación.

La competencia con referencia al producto principal de la empresa es fuerte (50\%), razón por la cual los empresarios realizan innovaciones especialmente a los productos, además de generar una relación más directa 
con los clientes, donde les facilitan el pago con plazos, o créditos, rebajan los precios, y, lo más importante, la ubicación de sus clientes que permite una comunicación y control constante entre el proveedor-productorcliente. El 22\% consideran que la competencia es débil, por el grado de innovación y calidad de sus productos.

Más de la mitad (56\%) de las empresas entrevistadas afirman no conocer los programas de gobierno que incentivan el desarrollo tecnológico, la investigación y la innovación de los cuales sólo el $25 \%$ han accedido. Es necesario impulsar a través de medios de información más efectivos, los programas que apoyan el avance productivo a través de la tecnología, más en estos sectores (alimentos y confección) que han sido tradicionales y que requieren de un cambio positivo hacia el conocimiento y el mejoramiento de la tecnología.

Uno de los mecanismos para lograr mayor apropiación y aprendizaje productivo de las empresas es la capacitación del personal. El recurso humano de toda empresa debe canalizar acciones en vía del mejoramiento de las competencias laborales asociadas al manejo de la información y el conocimiento. Aunque esto sea un objetivo primordial de los gobiernos territoriales y las empresas, se encuentran ciertas limitaciones, en párrafos anteriores se mencionaba la ausencia de las instituciones y la tendencia de las empresas a llevar procesos productivos individuales, las limitaciones tienen que ver con esto, predominantemente, se encuentra el poco acceso a las capacitaciones por altos costos (29\%), la resistencia al cambio de algunas empresas (17\%) y personal calificado insuficiente (16\%).

La introducción de las TIC's ha traído beneficios a las empresas; los empresarios destacan, en primer lugar, el aumento de la productividad (50\%), en segundo lugar el mejoramiento de la comunicación con los proveedores y clientes (33\%), en tercer lugar la eficiencia y rapidez en el proceso de producción (16\%). Esto indica que un enfoque hacia el manejo de la información adecuado y la comunicación permanente son esenciales para el desarrollo empresarial, no basta con tener un proceso de producción sino se encuentra articulado con gestiones empresariales encargadas de fortalecer las redes productivas $(61 \%$ de las pymes entrevistadas han hecho modificaciones en TIC's)

De la misma forma, más de la mitad de las empresas entrevistadas (61\%) realizan los cursos de capacitación sobre TIC's al interior de la empresa, esto indica que los mecanismos de capacitación y apropiación del conocimiento son internos, en gran medida posibilita mayor comunicación entre los trabajadores de la empresa y socialización de experiencias e información requerida para mejorar los procesos. (El $31 \%$ los realizan en otra institución y el $8 \%$ no responde)

Para las empresas entrevistadas es de gran importancia el desarrollo hacia la tecnología, lo ven como una posibilidad para abrir puertas en el mercado por medio de la innovación en los productos, otras para el mejoramiento de la calidad del producto, el cuidado del medio ambiente y fortalecimiento de procesos de producción. Algunas empresas opinan que por ser productos naturales, no requieren de procesos tecnológicos, o porque encuentran en la copia de productos la mejor fuente de rentabilidad.

Las incursiones hacia la biotecnología para las empresas del sector alimentos o el diseño para las empresas de confección, son diversas, se han creado áreas de diseño o estudio para mejoramiento de productos, se ha capacitado o contratado nuevo personal calificado, se ha realizado compra de software, investigaciones conjuntas con universidades e institutos y participación en ferias para la actualización de información y la creación de contactos.

Los avances hacia la biotecnología o al diseño han sido notorios en aspectos relacionados con el número de ventas, la conquista de nuevos mercados, la innovación de procesos y productos, el mejoramiento de la calidad, de los tiempos de producción. Esto indica que los sectores se han posicionado a partir de las pymes que desarrollan procesos tecnológicos, esto genera mayor sostenibilidad en el territorio.

Los empresarios opinan que los elementos para acceder a la biotecnología o al diseño son tener capital para poder invertir, realizar investigaciones donde el conocimiento se socialice y se generen procesos de apropiación en las empresas y por ende mayor eficiencia, espacios para realizar dichas investigaciones (como por ejemplo, laboratorios), maquinaria y equipos especializados y capacitación del personal. 
La necesidad de capital ha sido elemento muy reiterativo en las respuestas de los empresarios, los costos de inversión en tecnologías son altos y son pocas las posibilidades para acceder a ellas de manera independiente, por lo general las empresas ya consolidadas son las que pueden acceder a ellas ya que cuentan con un capital propio.

Los cambios o modificaciones realizadas a partir de la introducción de TIC's tienen que ver con la actualización y manejo de software especializado para diferentes áreas (diseño, contabilidad, entre otros), instalación de Internet y redes internas de información. Como se observa, se ha incursionado en gran medida el uso de software especializado, esto requiere de personal capacitado que se adapte a las constantes transformaciones y a los retos que los avances tecnológicos imponen en la actualidad.

Para el análisis factorial del presente modulo, se realizaron varias mediciones que hacen referencia a conjuntos de variables caracterizantes de los sujetos y que se relacionan entre sí. El primer grupo de variables, son los que hacen referencia a la relevancia del uso de las tradicionales como las nuevas tecnologías de información y comunicación TIC's. En estos resultados, se observa que un grupo de empresas predominante, se concentra en el uso de las nuevas TIC's, anteriormente se observaban los beneficios que éstas traen a las empresas, además de dichos beneficios específicos, la era de la globalización y la sociedad red exigen que los individuos se encuentren en comunicación constante, lo que obliga a las empresas a realizar transformaciones en los mecanismos que utilizan para sus procesos de comunicación y de gestión.

\section{Modulo redes industriales}

La economía informacional es una forma de organización social en la que el procesamiento y transmisión de la información se convierten en fuentes fundamentales de productividad y poder. Se basa en la producción inducida por la innovación para generar riqueza y para apropiársela de forma selectiva y a la vez depende de la capacidad del conocimiento y de la información para actuar sobre éste, lo que a su vez conforma una red ${ }^{5}$. Se dice que la sociedad en red no se trata sólo del espacio virtual, sino también de una serie de redes sociales configuradas en los espacios que comprenden nuestros domicilios, barrios, ciudades, estados-nación, construidas sobre principios de identidad nacional, étnica, cultural, de género, religiosa, etc. (CARDONA, et. Al., 2006, P. 15).

La economía del conocimiento requiere de articuladores. Para Castells (1996), las redes son el elemento fundamental del que están y estarán hechas las nuevas organizaciones. La globalización ha impuesto que estas estructuras flexibles conformadas en redes internas deban ser capaces de construir un modelo organizativo y productivo compatible con las redes productivas mundiales; así, se conforman nodos entrelazados que articulan una red que se soporta en la era informacional ${ }^{6}$.

El presente modulo hace referencia al fortalecimiento y construcción de redes productivas y cómo las TIC's pueden facilitar la comunicación en la cadena productiva y en el territorio. Más de la mitad de las empresas entrevistadas (61\%) pertenecen a un tipo de organización o gremio, con cobertura nacional (31\%), esto permite que haya una interacción de los factores geográficos y sectoriales. Utilizan las TIC's para generar procesos de comunicación más eficientes.

\footnotetext{
${ }^{5}$ La organización se genera alrededor de centros de mando y control, capaces de coordinar, innovar y gestionar las actividades entrecruzadas de las redes empresariales y de los países desarrollados y en proceso de serlo. En efecto, constituyen redes de producción y gestión, cuya flexibilidad no necesita incorporar trabajadores y proveedores, sino tener capacidad de acceso a ellos cuando convenga y en el momento y cantidades requeridos en cada caso particular.

${ }^{6}$ De esta forma surgen conceptos como outsourcing, alianzas estratégicas, subcontratación, teleinformática, que sustentan la sociedad moderna y las estructuras productivas actuales. La capacidad de conexión de las firmas, no sólo al interior de su estructura, sino también con sus proveedores, con sus clientes, con las instituciones gubernamentales, con el sistema financiero, con la academia y con las redes mundiales es la condición para que las estructuras productivas pequeñas, medianas o grandes tengan la oportunidad de posicionarse en el mercado ${ }^{6}$. Se debe sumar la necesidad de las certificaciones, la diversificación y la especialización, el encadenamiento productivo intramercados, redes verticales y las potencialidades del territorio en donde se encuentra asentada la firma y que posibilite un mayor ciclo de vida. La gestión del desarrollo a través de redes, acumula conocimiento en el territorio.
} 
El $32 \%$ de las empresas entrevistadas comparten información sobre los clientes con otras empresas o asociaciones, seguido de información sobre los clientes. Entre mayor confianza y pertenencia se tenga en la red, mejores son los procesos internos de las empresas, ya que se comparten formas de hacer las cosas, además de posibles contactos para comercializar o para proveer con una mejor calidad o precio.

Dado el aprendizaje colectivo, los acuerdos han mejorado la oportunidad con que se atiende a los clientes (50\%), aumentan los segmentos de producción que pueden cubrir dentro del ramo (30\%) y mejorar la calidad del producto frente a otros competidores (20\%). También se han realizado adaptaciones empresariales a partir de dichos acuerdos ( $41 \%$ de las empresas entrevistadas). Sin embargo, son pocas las empresas que han establecido alianzas estratégicas para producir o comercializar los productos. De las pocas empresas que realizan dichas alianzas (33\%) sobresale los siguientes motivos: compras a través de intermediarios (26\%), ventas a través de distribuidores (22\%).

La concentración de las empresas se da hacia los dos factores, predominando los que pertenecen a una red hacia el factor uno y las empresas que no tienen red hacia el factor dos. Sin embargo, encontramos una baja pertenencia a redes dentro de las empresas entrevistadas, esto puede disminuir las posibilidades de articulación para el aprendizaje productivo a nivel territorial y sectorial.

\section{Módulo conocimiento y competencias laborales}

El paradigma de la tecnología y la información supone una transformación en el seno de la sociedad. Una sociedad cognitiva, que genera niveles altos de terciarización de su actividad económica necesitará cambios en las políticas de reclutamiento y selección de trabajadores con las competencias laborales requeridas por la transición de los modelos de producción fordista hacia modelos organizativos más flexibles, como los que exige en la actualidad el mercado

La definición de las competencias, y obviamente su aprendizaje, exigen entonces acuerdo y colaboración entre el mundo de la educación y el mundo del trabajo, se adquieren en trayectorias que implican una combinación de educación formal, aprendizaje en el trabajo y, eventualmente, educación no formal. La capacitación específica se construye sobre esta base de competencias adquiridas en la escolaridad formal y la experiencia. La formación profesional entonces debe estar focalizada en familias específicas de ocupaciones en el mundo del trabajo integrando las competencias como comportamientos efectivos con las habilidades necesarias para el desempeño de las tareas ocupacionales, el uso del equipamiento y la tecnología, y el aprendizaje organizacional de las empresas y mercados.

El modulo conocimiento y competencias laborales hace referencia a la manera como las empresas desarrollan procesos de aprendizaje y capacitación involucrando los diferentes actores del proceso productivo y acciones encaminadas hacia el conocimiento y el mejoramiento del proceso de trabajo y del funcionamiento de la empresa en general.

Los conocimientos o habilidades desarrollados por las empresas entrevistadas son los relacionados con conocimientos sobre procesos de trabajo (20\%), conocimiento sobre administración y gestión (20\%), conocimiento sobre manejo de maquinaria para la producción (20\%), conocimiento sobre diseño de productos (19\%), conocimiento sobre mercadeo (14\%) y por último conocimiento sobre TIC's (7\%). Esto manifiesta una fuerte necesidad de calificación por parte de los trabajadores, pero al mismo tiempo muestra la apropiación de los procesos de gestión del conocimiento por parte de las empresas entrevistadas. Más de la mitad de los empresarios entrevistados (58\%) afirman no tener dificultades para cubrir los puestos de trabajo de mayor competencia laboral. En las empresas, se han dado procesos de capacitación al personal vinculado (66\%), lo que genera apropiación y una mayor construcción del conocimiento colectivo que beneficie a la empresa. Esta capacitación tiene diversos mecanismos, puede ser interno (44\%), externo (11\%) o ambas (14\%).

Aunque las empresas corren el riesgo de retiro del personal capacitado (50\%), consideran que es mejor realizar capacitaciones para la actualización del conocimiento y mayor eficiencia en la producción. La socialización de los conocimientos para la producción en las empresas es corto, debido a que, desde el principio, las empresas definen un perfil de trabajador con conocimientos previos y experiencia. 
De las empresas que no ofrecen capacitación, el $31 \%$ afirman que no acceden a ella por los altos costos que implica. El reto de los gobiernos regionales y de las organizaciones gremiales es realizar capacitaciones sectoriales que permitan la sistematización de experiencias empresariales e intercambio de saberes, además de procesos de transferencia de tecnología viables. También, se requiere del compromiso con las empresas para integrar procesos que beneficien no solo al interior sino al territorio. Esto requiere no sólo de inversión de capital sino de tiempo.

En un contexto en el que se tiende a la flexibilización, en un proceso de cambio tecnológico y organizacional por efecto de la reestructuración productiva, donde la subcontratación entre empresas grandes y pequeñas se vuelve común, donde la polivalencia y la rotación de ocupaciones se convierten en habituales, la lógica de las competencias ha pasado al centro de la escena en las empresas, alcanzando nuevas formas de reclutamiento, promoción, capacitación y remuneración. El foco de atención se ha desplazado así de las calificaciones a las competencias, es decir, al conjunto de saberes puestos en juego por los trabajadores para resolver situaciones concretas del trabajo.

La noción de competencia, tal como es usada en relación al mundo del trabajo, se sitúa a mitad de camino entre los saberes y las habilidades concretas; la competencia es inseparable de la acción, pero exige a la vez conocimientos razonados, ya que se considera que no hay competencia completa si los conocimientos teóricos no son acompañados por las cualidades y la capacidad que permita ejecutar las decisiones que dicha competencia sugiere. Son entonces un conjunto de propiedades en permanente modificación que deben ser sometidas a la prueba de la resolución de problemas concretos en situaciones de trabajo que entrañan ciertos márgenes de incertidumbre y complejidad técnica.

\section{Consideraciones finales}

Las dimensiones propuestas para la investigación son los campos problemáticos del desarrollo regional e industrial que están asociadas a relaciones que apoyan, por un lado, la competencia, la eficiencia y las formas de intercambio; y por el otro, la cooperación y la solidaridad, en una nueva perspectiva del concepto de competitividad, que toma en cuenta la importancia del tiempo y del espacio en la generación de propuestas de política de desarrollo regional. Además, el papel de los gobiernos nacionales y locales adquiere importancia en la medida en que las regiones, y en ellas las firmas, enfrentan un período de transición complejo hacia un nuevo modelo de desarrollo, en el cual tienen que globalizarse y priorizar las políticas dirigidas a la competitividad, a la educación, a la inversión privada, a las exportaciones, a la conectividad y a la convivencia.

El cambio a una economía del conocimiento trae aparejado un aumento de las oportunidades para los negocios tanto en el ámbito nacional como internacional. Esto se debe al papel que cumple el 'know how', el surgimiento de nuevos mercados y las capacidades de gestión de la información de las empresas resultante de la expansión de las TIC's. Han aparecido nuevos mercados y ha mejorado el acceso a los mismos a través de la creación de redes más eficientes de producción y por lo tanto, han empezado a cambiar los precios relativos (y las oportunidades competitivas).

Las ventajas de este cambio parecen estar dirigidas hacia las empresas con mayores capacidades para generar o capturar el mayor valor agregado, las marcas más conocidas y la administración más efectiva del conocimiento para adaptarse a mercados en evolución. La competencia entre proveedores se ve fomentada por el mayor tamaño de los mercados y está asociada al uso de las TIC's.

La sociedad contemporánea cada vez más está basada en la transmisión y uso del conocimiento y de la información, donde la capacidad innovadora de las firmas en su proceso competitivo desempeña un nuevo papel, y se caracteriza por un fuerte crecimiento del trabajo cognitivo y relacional (LA ROVERE, et.al., 2000).

La innovación y el aprendizaje se convierten en procesos fundamentales para generar ventajas competitivas en el ámbito de la nueva economía. En el pasado, la mayoría de las organizaciones existían para fines económicos específicos de forma que la innovación y el aprendizaje no formaban parte de sus objetivos. No 
obstante, varios autores, estudiosos de la creación del conocimiento en las organizaciones, destacan su importancia creciente como recurso estratégico.

Dentro de los factores fundacionales también se observan algunos que hacen referencia a la influencia que tiene el mercado, el sector, el apoyo de entidades públicas y privadas, la competencia inexistente en el mercado por poseer productos innovadores adecuados a necesidades no satisfechas aún. En esta medida se puede determinar que una de las necesidades prioritarias en las empresas es el desarrollo y conocimiento tanto del producto como del proceso, pero también del entorno que la rodea y las posibilidades de crear innovación que integre la experiencia de los empresarios.

Así como se presentan posibilidades, también se presentan obstáculos para la creación de empresas. Los empresarios entrevistados manifiestan falta de recurso humano asociado a la poca experiencia y conocimiento, por un lado de las habilidades requeridas para la producción y por el otro de las condiciones en las que se encuentra el mercado y el sector. Además se presentan obstáculos por trámites burocráticos que hacen referencia a costos y normatividad, obstáculos por el mercado, asociado a la alta competencia, obstáculos por falta de clientes, por falta de acceso a tecnología, entre otros.

Las empresas entrevistadas se caracterizan por pertenecer a dos eslabones de la cadena productiva, producción y comercialización; en la producción, lo que determina el comportamiento de las empresas con respecto al tipo de tecnología necesaria, si la empresa es productora (la mayoría -62\%-de las empresas son productoras), necesitaría de tecnologías de producción, software especializados, maquinaria dura, mientras que en los proveedores o distribuidores requerirían de uso de TIC's y de permanente procesos de comunicación con los clientes y demás miembros de la cadena. Pero, dada la importancia atribuida al uso de TIC's, las empresas productoras, proveedoras y comercializadoras se enfocan hacia un conocimiento y un manejo de éstas en pro de la eficiencia y el buen funcionamiento de la empresa.

Las empresas entrevistadas tienen unas características que dan cuenta de las transformaciones estructurales enfocadas hacia el aprendizaje y los nuevos retos que suponen la economía del conocimiento, relacionadas con los siguientes elementos: 1) Las competencias laborales asociadas al conocimiento de tecnologías de producción y TIC's. 2) La localización como factor determinante para las decisiones de la empresa en materia de transferencia tecnológica y establecimiento de relaciones industriales.3) El desarrollo de procesos empresariales que tienen en cuenta no solo el proceso de trabajo sino la investigación sobre productos, eficiencia y calidad en el proceso. 4) El mejoramiento de la comunicación entre las empresas que conforman redes o alianzas. 5) La introducción de los procesos empresariales hacia la biotecnología o el diseño. 6) El poco apoyo de las instituciones gubernamentales y privadas para la transferencia tecnológica. 7) La innovación como objetivo de las empresas, la inserción y estabilidad en el mercado a partir de los aprendizajes al interior de la empresa. 


\section{Bibliografía.}

BARCELÓ LLAUGER, María Hacia una economía del conocimiento. Madrid. ESIC Editorial, 2001.

BIANCO, Carlos; LUGONES, Gustavo (coord.); PEIRANO, Fernando; SALAZAR, Mónica. Indicadores de la sociedad del conocimiento e indicadores de innovación vinculaciones e implicancias metodológicas y conceptuales. Seminario Internacional "Redes, tic's y desarrollo de políticas públicas" UNGS-EGIDA Firenze Buenos Aires, 2002

BOSCHERINI, Fabio; NOVICK, Marta y YOGUEL, Gabriel (compiladores) Nuevas tecnologías de la información y comunicación: los límites de la economía del conocimiento. Buenos Aires: Miño y Dávila SRL, 2003.

CANO, Carlos Andrés. La Sociedad Informacional: La nueva estructura de poder social, político y económico. Medellín. Universidad EAFIT, 2006.

CARDONA ACEVEDO, Marleny; ANGEL, Adriana. Impacto de las redes industriales en la política sectorial: El caso de los alimentos, textil-confección y metalmecánica. Universidad EAFIT, 1999

CARDONA ACEVEDO, Marleny; RAMÍREZ, Mauricio; ACEBEDO, Carlos Alberto; VERA ACEVEDO, Luz Dinora. (Sociedad en red, Innovación y sistemas de innovación: Análisis de caso de las telecomunicaciones y el Software para la industria en Colombia en Cinco ciudades: Medellín, Manizales, Bogotá, Cali y Barranquilla. Medellín: Universidad EAFIT, 2003.

CASTELLS, Manuel. "La sociedad red. La era de la información: Economía, sociedad y cultura". Alianza Editorial, Madrid, 1996

CHAPARRO, Fernando. "Haciendo de Colombia una Sociedad del Conocimiento. Conocimiento, innovación y construcción de sociedad: una agenda para la Colombia del siglo XXI ". Tm editores/ Colciencias. Santafé de Bogotá, 1998

CHAPARRO, Fernando.. Apropiación Social de las Nuevas Tecnologías de la Información y las Comunicaciones (TICs) e Informatización de la Sociedad Colombiana. Corporación Colombia Digital, Bogotá, 2004

DAHLMAN Erik B.; Vu-Chuen Jou. IEEE,. Evoling technologies for 3G cellular wireless, Communications Magazine, Feb2006, Vol. 44 Issue 2, p62-64, 2p; (AN 19983619), 2000

DAVID, Paul A. y FORAY, Dominique. Una introducción a la economía y a la sociedad del saber. En Revista internacional de ciencias sociales. Número 171. La sociedad del conocimiento, 2002

DEL VALLE CABALLERO, Jaime). Economía del conocimiento. Unos apuntes necesarios. http://www.economistaspr.org/c2006/ponencias2006/Del_Valle.pdf. 2006

DOSI, G. Y MALERBA, F. "Organizacional Learning and Institucional Embeddedness", en Organitation and Strategy in the Evolution of Enterprise. Ed. Dosi y Malerba (Pag.1-16), 1996.

DRUCKER, Peter. La sociedad post capitalista. El conocimiento, su economía; su productividad. Bogotá: Norma, 1994

EDGINGTON D. Learning Regions: Lessons for Developed and Developing Countries, Paper presented at the Global Forum on Regional Development Policy, UNCRD, Dec., Nagoya, Japan, 1998.

HANSSON, Bo. Company based determinants of training and the impact of training on company performance, Personnel Review, Vol. 36 Issue 1/2, p311-331, 21p; (AN 24466897), 2000

HOWITT, Peter, Monetary Policy and the Limitations of Economic Knowlodge Post Walrasian Macroeconomics: Beyond the Dyanamic Stochastic General Equilibrium Model, edited by David Colander. New York: Cambridge University Press, 347-67, 1996

ELSTER, J. "El cambio tecnológico: Investigación sobre la racionalidad y la transformación social". Ed. Gedisa. Barcelona, 1990 
LA ROVERE, Renata Lèbre y HASENCLEVER, Lía. Innovación, competitividad y adopción de tecnologías de la información y de la Comunicación en pequeñas y medianas empresas: algunos estudios de caso sobre Brasil En: Boscherini, Fabio; Novick, Martay Yoguel, Gabriel (compiladores). Nuevas tecnologías de la información y comunicación: los límites de la economía del conocimiento. Buenos Aires: Miño y Dávila SRL, 2003.

MUNSTER INFANTE, Blanca. Capitalismo y la economía del conocimiento. Seminario Nacional de Intercambio científico. Talento y capital Humano. GECYT, 2007.

NELSON, RICHARD. "National Innovation Systems: A retrospective on a Study. en Organitation and Strategy in the Evolution of Enterprise. Ed. Dosi y Malerba, 1996.

OCDE. "La innovación tecnológica: definiciones y elementos de base" Pág. 25-26; 35-44, 1996

PARADA, Nereida. XX. La universidad en la encrucijada de la sociedad del conocimiento y la cultura de la sustentabilidad. Disponible en: http://www.saber.ula.ve/cgi win/be_alex.exe?Documento=T016300003594/7\&term_termino_2=e:/alexandr/db/ssaber/Edocs/grupos/giecal/publicacione s/monografias/cuaderno2/capitulo6.pdf

PEREZ Yuste, Antonio. Desentrañando la sociedad de la información. Revista Mundo Electrónico No 339, 2003

Revista REDES. Volumen 3, Nro. 6. Universidad Nacional de Quilmes. Buenos Aires.

ROLDÁN, Martha. Una Historia Inconclusa: Conocimiento, Políticas de Ciencia y Tecnología, y Desarrollo en el Contexto de la Mundialización Contemporánea. Reflexiones Sobre el Caso Argentino (1990s-2000s). Disponible en: http://www.politicahoje.com/ojs/viewissue.php?id=5\#Dossi\%C3\%AAs, 2003.

SCHUMPETER, Josheph. Análisis del cambio económico. Ensayos sobre el ciclo económico. Ed. Fondo de cultura económica, México. Disponible en http://eumed.net/cursecon/textos/schump-cambio.pdf, 1935.

STEEDMAN, Ian. From Classical Economics to the Theory of the firm, Manchester School (14636786), Vol. 69 Issue 2, p227, 3p; (AN 6748217), 2001

VALLE FLÓREZ, Mónica María. Acercamiento teórico a la sociedad de la información: sistemas de producción y tecnología. Revista Anagramas. VOL. 3 No 5. Julio/Diciembre, 2004

YONEJI, Masuda, The opportunity society, Futurist, Sep/Oct 90, Vol. 24 Issue 5, p8-11, 4p, 3 illustrations, 4 bw; (AN 9009241575), 1990. 